\title{
Malignant Phyllodes Tumour in a Non-Governmental Health Centre (Faith Alive Foundation) in Jos, Plateau State North Central Nigeria. A Case Report and Review of Literature.
}

\author{
Silas Olugbenga ${ }^{1 *}$, Isichei Mercy ${ }^{2}$, Isichei Christian ${ }^{2}$ \\ ${ }^{1}$ Pathology Department, Jos University Teaching Hospital (JUTH) Jos, Plateau State, \\ Nigeria. ${ }^{2}$ Faith Alive Foundation Jos, Plateau State Nigeria \\ *Corresponding Author \\ Pathology Department Jos University Teaching Hospital Jos, Plateau State Nigeria \\ Structured Summary
}

\begin{abstract}
Malignant Phyllodes tumour is a rare breast tumour seen mainly in women though it has infrequently been reported in some men. Malignant phyllodes is rarer then its benign counterpart (Cystosarcoma Phyllodes) mainly occurring in $5^{\text {th }}$ decade and above though rarely in lesser decades. It has metastatic potentials with its attendant morbidity and mortality. This is the first we are seeing in our health facility, occurring in the $3^{\text {rd }}$ decade of life in a lady, with absence of sophisticated diagnostic facilities. A high index of suspicion is therefore required thus necessitating this report.
\end{abstract}

\begin{abstract}
Malignant Phyllodes tumour is a rare breast tumour seen mainly in women though it has infrequently been reported in some men. Malignant phyllodes is rarer than its benign counterpart (Cystosarcoma Phyllodes) mainly occurring in $5^{\text {th }}$ decade and above though rarely in lesser decades. It has metastatic potentials with its attendant morbidity and mortality. This is the first case in our health facility, occurring in the $3^{\text {rd }}$ decade of life in a lady. With absence of sophisticated diagnostic facilities, a high index of suspicion is therefore required thus necessitating this report.
\end{abstract}

Conclusion:- Malignant Phyllodes tumour though rare below $5^{\text {th }}$ decade, can however occur. In developing countries characterized by dearth of sophisticated diagnostic tools, a high index of suspicion is necessary.

Key words: - Phyllodes, Cystosarcoma phyllodes, Malignant.

\section{Introduction}

Phyllodes tumours of the breast are fibro epithelial neoplasms that have the potential for recurrence and metastasis ${ }^{(1)}$ Grading into benign, borderline and malignant categories is based on a constellation of histologic characteristics that include the degree of stromal hypercellularity, stromal cytologic atypia and mitotic activity, stromal overgrowth and circumscribed versus invasive margins. Although histological features have been helpful to some extent in predicting biologic behavior, specific parameters that can define the likelihood of recurrence are not universally accepted. Various investigators have found cellular pleomorphism, stromal overgrowth, tumour necrosis and heterologous stromal elements or a combination of these histologic features to be prognostically useful. On the other hand, several authors have concluded that adequacy of surgical margins is of paramount importance and that histological factors have an inconsistent influence on biologic behaviour ${ }^{(1)}$. Sarcomatous stromal elements including angiosarcomas, chondrosarcomas, leiomyosarcomas, osteosarcomas and rhabdomyosarcomas are rarely encountered in malignant phyllodes tumours. The finding of a malignant heterologous element places the tumour into a malignant category. Local recurrence of phyllodes tumour is not an indicator for malignancy as it has been described in benign, borderline and malignant phyllodes tumours ${ }^{(2)}$.

\section{Case Presentation}

MC is a 26 year old female student of a tertiary institution .She presented to our health facility with a 4months history of progressive left breast swelling. Patient was apparently well until six months prior to presentation when she noticed a painless, freely mobile lump in the upper outer quadrant of the left breast. She earlier had a lumpectomy at a Specialist Hospital four hours drive from our facility.Histopathological result of the excised lump at the Specialist Hospital revealed chronic inflammatory cells. Lump reappeared six weeks later at site of excision, this time growth was fast and within four months it had extended to involve three quadrants of the breast. No associated nipple discharge, ulceration or weight loss. No respiratory difficulties, chest pain or symptoms of 
other system involvement. Pain was dragging in nature as tumour size increased but later became excruciating with extensions of tumour over the medial part of left clavicle. At the time of surgery, one month after presentation, tumour had involved the whole breast. No family history of similar illness, no history of alcohol ingestion or cigarette smoking.

Examination shows a young lady not ill looking, mildly pale, no lymphadenopathy with a huge diffusely enlarged left breast with flat nipple and areolar, shiny overlying skin, and some lobulated areas. The mass had firm and cystic areas, tender at the upper inner quadrant areas where it was also fixed to the underlying structures and medial part of the clavicle. No lump in the contra lateral breast, no anxillary, supraclavicular or cervical lymph node enlargement.Liver, spleen was not palpably enlarged. No ascites.Chest was clinically clear.

Breast ultrasound showed multiple mixed echogenic masses of varying size, largest measuring $7.5 \times 10 \mathrm{~cm}$ and consisting of many smaller masses fused together. Ultrasound guided aspiration of cystic areas was of altered blood.

Chest X-ray and ECG were essentially normal. Apart from a haemoglobin value of $10 \mathrm{~g} / \mathrm{dL}$, all other laboratory investigations were essentially normal including HIV, Hepatitis B and C screenings.

Biopsy -FNAC (Fine Needle Aspiration cytology) was reported as phyllodes Tumour as well as the trucut biopsy which showed the same result.

Right breast showed normal findings. Simple mastectomy was done with wide margin of excision and primary skin closure. Mass sent for histology and reported was Malignant Phyllodes tumour (Malignant Cystosarcoma Phyllodes). Patient was later referred to Ahmadu Bello University Teaching Hospital, Zaria, Kaduna State, Nigeria for radiotherapy. She is presently responding well to management.

\section{Gross / microscopic description}

Gross Specimen (mastectomy) shows a huge breast weighing $2.2 \mathrm{~kg}$ and measuring $21 \mathrm{~cm}$ by $19 \mathrm{~cm}$ by $16 \mathrm{~cm}$.Nipple is flat and there is no ulceration and no adjoining lymph nodes. Cut surface shows a grayish yellow surface with few cystic cavities. Hemorrhagic areas are also observed.

Histologic section shows a stratified sqamous epithelium overlying a highly cellular Stroma in which is seen spindle shaped cells with elongated nuclei and bipolar cytoplasm processes. They are arranged in sheets and fascicles and most exhibit hyperchromasia, atypia and increased mitosis most of which are abnormal. Elsewhere is seen compressed ductular structures lined by basophilic epithelial cells. Adipocytes and thick walled blood vessels are also observed.

\section{Review of Literature}

Although phyllodes tumour may have been described as early as 1774 , the lesion was first fully characterized in 1838 by Johannes ${ }^{(2)}$.The term cystosarcoma phyllode was chosen to emphasize the leaf like pattern and fleshy appearance of the lesion. Among the many other names subsequently applied to the tumour, the only other term currently widely used are periductual stromal tumour and phyllodes tumour. The former term was put forth to emphasize putative origin from specialized periductal stoma. Use of the term phyllodes tumour is preferable to avoid a diagnosis of sarcoma for benign variants. Pathogenesis is said to be genetic based on inactivation of X-chromosome - linked androgen receptor (AR) gene. Phyllodes tumours are usually solitary and unilateral. Rarely, multifocal phyllodes tumour has been detected in a single breast it is very rare in men.

Malignant phyllodes occasionally occur following a previous fibroadenoma or on its own. It is characterized by rapidity of growth and common in the $5^{\text {th }}$ and $6^{\text {th }}$ decades of life as opposed to its benign counterpart which occurs more in the $3^{\text {rd }}$ and $4^{\text {th }}$ decades of life ${ }^{(3)(4)}$.Malignant phyllodes tumours of the breast is rare all over the world ${ }^{(5)}$.In the western world, studies have show a very low incidence mainly between $0.3-0.9 \%{ }^{(5)}{ }^{(6)}$. This is consistent with African studies as reports shown incidence ranging between $0.3-0.5 \%{ }^{(7)(8)}$. Obafunwa J.O et al in the late 80 's reported only 2 cases $(0.3 \%)$ of malignant phyllodes in a review over a 9 year period in Jos ${ }^{(8)}$. Irabor D.O et al in Ibadan showed probability of malignancy to increase with age greater than $5^{\text {th }}$ decade ${ }^{(7)}$. Wide excision is preferred to a local one in surgical management of malignant phyllodes tumour as recurrence rates are high for borderline $(40 \%)$ and malignant phyllodes $(65 \%)$ after local excision of tumour but low (borderline $(29 \%)$, malignant (36\%) after a wide excision ${ }^{(9)}$.

Molecular diagnostic techniques are recently being used to diagnose malignant phyllodes tumour as they are said to express a proto-oncogene c-kit which encodes a tyrosine kinase receptor (CD117) and is a marker for gastro intestinal tumour (GIST) (9) 


\section{Discussion}

Malignant phyllodes tumour can also occur in less than $5^{\text {th }}$ decade of life. The earlier histology report of chronic inflammatory cells for the lumpectomy specimen in this index case suggests a malpractise as histologic features of any proliferative breast lesion is usually obvious, though immunohistochemistry which is absent in our environment could have helped this initial d i a g n o s i s. Th e d e a $\mathrm{rth}_{\mathrm{o}} \mathrm{f}$ specialists(histopathologists) and modern diagnostic tools is still an issue that needs urgent attention in our setting as wrong diagnosis with resultant mismanagement is a recurring decimal.Government should as a matter of urgency discourage the exodus of medical doctors and mordernize our health facilities to halt this negative trend.

\section{Conclusion}

A high index of suspicion, use of histopatologists and availability of modern diagnostic tools is necessary for early diagnosis and management of malignant phyllodes tumour.

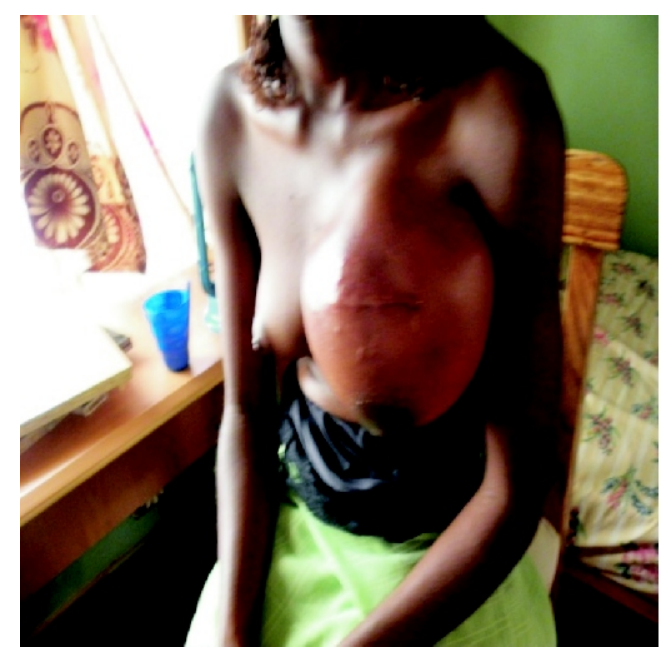

Patient before surgery with Biopsy scar Left breast

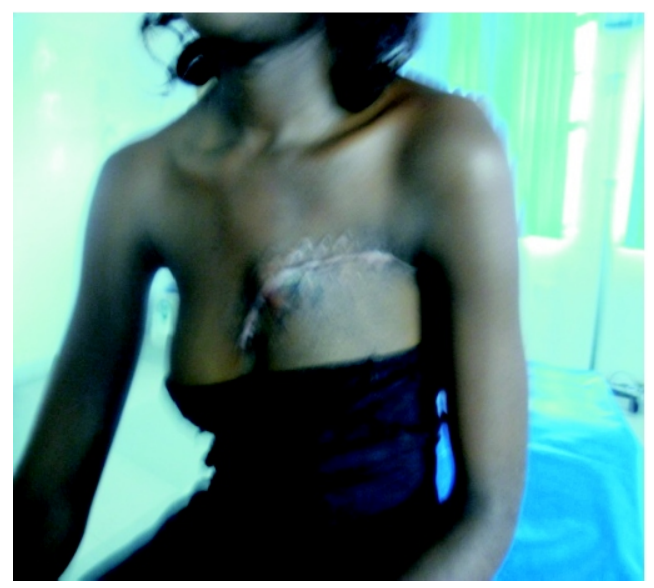

Patient before surgery with extended Biopsy scar left breast

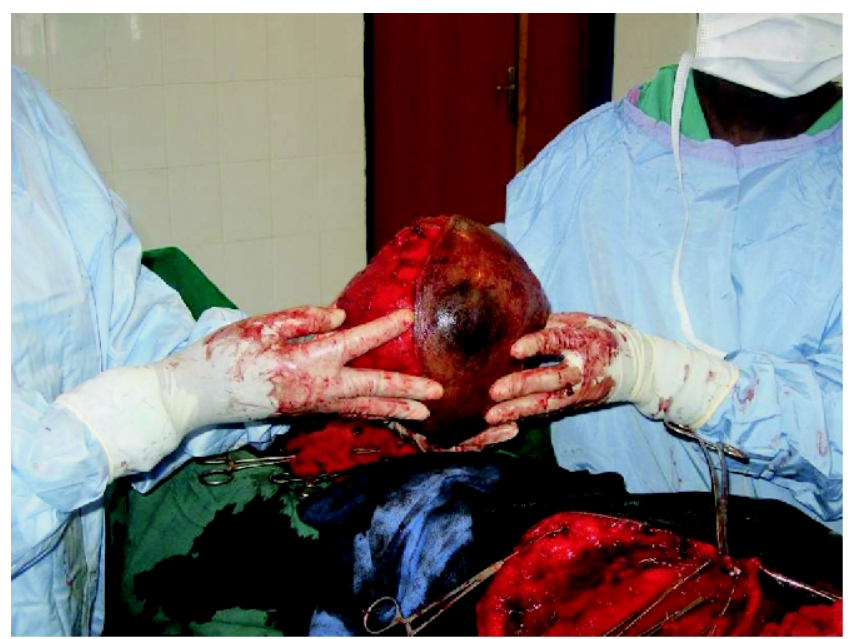

Left breast tumour being removed during surgery

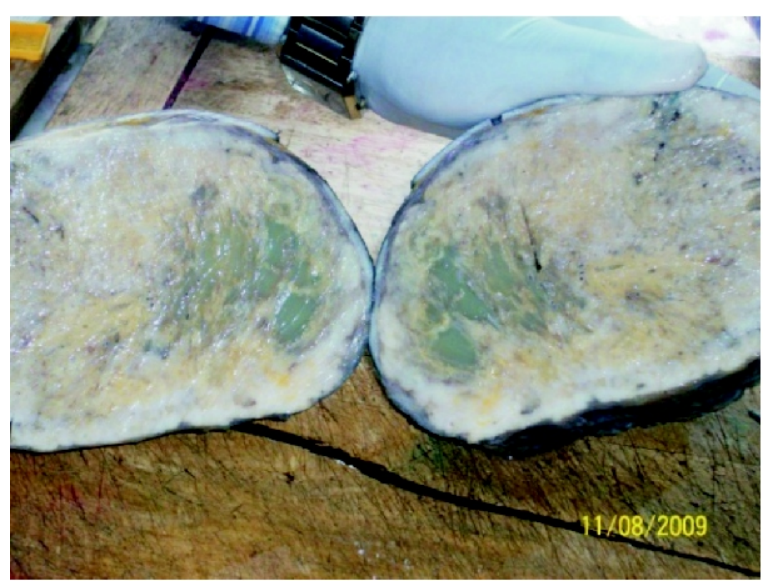

Cut section of breast showing cystic areas in a yellowish-gray fibrofatty stroma. Weight of breast is $2.2 \mathrm{~kg}$ and measures $21 \mathrm{~cm}$ by $19 \mathrm{~cm}$ by $16 \mathrm{~cm}$.

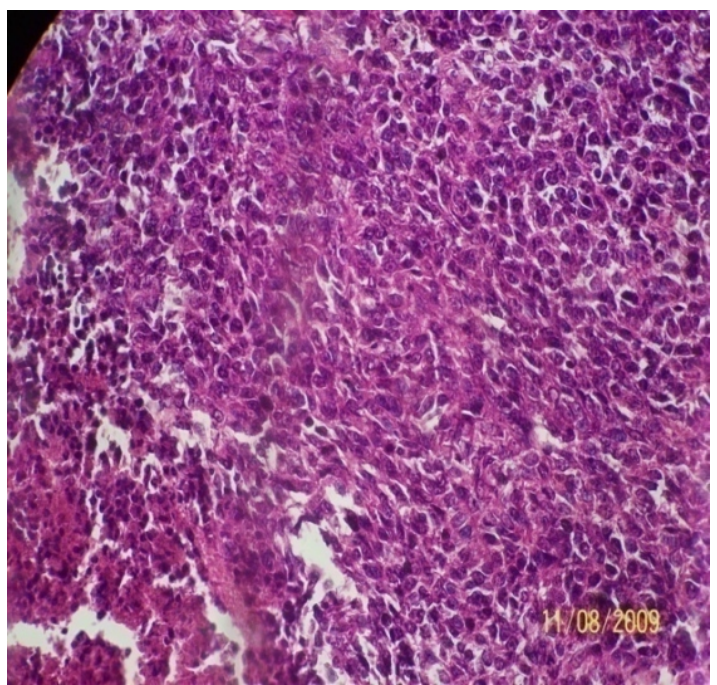

Magnification $\mathrm{x} 40$

Stain $\mathrm{H} \& \mathrm{E}$

Histologic section shows neoplastic spindle shapped stromal cells exhibiting hyperchromasia, pleomorphism and high nucleo cytoplasmic ratio. Areas of attendant necrosis is also observed in the lower left portion of the diagram. 


\section{References}

1. Rhodes R, Frankel K, Davies R, Tatter D. Metastatic Cystosarcoms Phyllodes. A report of 2 cases presenting with Neurological Symptoms. Cancer 1978, 41: 1179 1187.

2. Turalba C, El-Mahdi A, ladaga L. Fatal metastatic Cystosarcoma Phyllodes in an Adolescent Female. Case report and review of treatment approaches. $\mathrm{J}$ of Surg Oncol 1986; 33: 176181.

3. Treves N, Sunderland D.A. Cystosarcoma Phyllodes of the breast: A malignant and a benign tumour. Cancer. $1951 ; p_{g} 12861291$.

4. Guerrero M.A, Ballard B.R, Grau A.M. Malignant phyllodes tumour of the breast: review of literature and case report of stromal overgrowth. Surg Oncol 2003; 12 (1):27-37.
5. Arcuri M.F, Del Rio P, Martella E.M et al. Giant Malignant Phyllodes tumour: case report. G chir. 2007; 28 (6) 251252.

6. Carter B.A, Page D.L Phyllodes tumours of the breast: local recurrence versus metastatic capacity. Human pathol. 2004; 35: 10511052.

7. Irabor D.O, Okolo C.A. An audit of 149 consecutive breast biopsies in Ibadan, Nigeria Pak J Med Sci 2008;24(1):2.

8. Obafunwa J.O, Nwana EJ. Malignant cystosarcoma phyllodes: report of two cases. Cent Afri J med 1989; 35 (10): 510513

9. Histologic features predict local recurrence after breast conserving therapy. Breast Cancer Res. Treat. 1999;57 (3): $291-295$ 